\section{Corrective surgery for canine patellar luxation in 75 cases (107 limbs): landmark for block recession}

\author{
Mitsuhiro Isaka, Masahiko Befu, Nami \\ Matsubara, Mayuko Ishikawa, Yurie \\ Arase, Toshiyuki Tsuyama, Akiko Doi, \\ Shinichi Namba \\ Marble Veterinary Medical Center, \\ Fujisawa, Japan
}

\section{Abstract}

Canine medial patellar luxation (MPL) is a very common orthopedic disease in small animals. Because the pathophysiology of this disease involves various pathways, the surgical techniques and results vary according to the veterinarian. Further, the landmark for block recession is not completely clear. We retrospectively evaluated 75 dogs (107 limbs) with MPL in whom our landmark for block recession was used from July 2008 to May 2013. Information regarding the breed, age, sex, body weight, body condition score (BCS), lateral $v s$ bilateral, pre-operative grading, surgical techniques, removal of implants, concomitance with anterior cruciate ligament (ACL) rupture, re-luxation, re-operation, and rehabilitation was obtained from the medical records. The breeds were as follows: Chihuahua $(\mathrm{n}=23)$, Pomeranian $(\mathrm{n}=12)$, Yorkshire Terrier ( $n=9)$, and so on. The study group consisted of 33 males (castrated $n=13$ ) and 42 females (spayed $n=21$ ). The median age was $53.3 \pm 35.9$ months (32-146 months); 13 cases were less than 12 months of age (17.3\%). The pre-surgical BCSs were as follows: $1(\mathrm{n}=0), 2$ $(n=20), 3(n=24), 4(n=24)$ and $5(n=7)$. The body weight was $4.51 \pm 3.48 \mathrm{~kg}(1.34-23.0 \mathrm{~kg})$; 71 cases $(94.7 \%)$ were less than $10 \mathrm{~kg}$. The MPL grades (each limb) were G1 $(n=1), G 2$ $(\mathrm{n}=18)$, G3 $(\mathrm{n}=78)$, and G4 $(\mathrm{n}=10) ; 32$ cases were bilateral and 43 cases were unilateral (right $\mathrm{n}=27$; left $\mathrm{n}=16$ ). The specific surgical procedure (distal femoral osteotomy) was 3 stifles in Chihuahuas. Concurrent with ACL rupture was 16/107 stifles (15.0\%) corrected with the over-the-top method or the extracapsular method in Papillons (5/6), Chihuahuas (5/23), and so on. The occurrences of re-luxation and re-operation were 3 out of 107 stifles (2.8\%) and $0 \%$, respectively. In this retrospective study, we present a potentially good surgical landmark for block recession of MPL in dogs.

\section{Introduction}

Medial patellar luxation (MPL) is a common orthopedic disease in dogs, and this condition accounts for many of the corrective surgeries that are performed in dogs. Consequently, several studies have reported the outcomes after corrective surgery for MPL in dogs.1-5 The outcome has generally been considered in terms of either post-operative limb function or the development of osteoarthritis. However, few studies have examined in detail the incidence and the types of complications after corrective surgery for patellar luxation. A recent study reported that the complication rate after surgery is $18 \%$ and the occurrence of re-luxation requiring re-operation is $8 \% .{ }^{1}$ However, these variables may depend on the experience of the surgeon, breed distribution, and several other factors.

Sulcoplasty deepens the trochlear groove and reportedly reduces patellar instability, and these effects have been confirmed in canine stifles. ${ }^{1}$ Femoral trochlear sulcoplasty significantly reduces the frequency of MPL, suggesting that femoral trochlear sulcoplasty should be performed as part of MPL surgery to minimize the risk of MPL. In a comparison between trochlear block recession (TBR) and trochlear wedge recession (TWR) for trochleoplasty in canine patellar luxation using a cadaver model, TBR increased the proximal patellar depth and the patellar articular contact with the recessed proximal trochlea, recessed a larger percentage of the trochlear surface area, and resulted in a greater resistance to MPL in an extended position as compared with TWR. TBR may help limit the development of stifle degenerative joint disease in dogs treated for canine MPL.5-7 Further, rectangular recession trochleoplasty for treatment of MPL in dogs has been well described. ${ }^{8}$ However, no landmark has been established for the surgeon during procedure. The aims of this study were as follows: to review the medical records of dogs that had corrective surgery for MPL, to compare the descriptive epidemiology with previous reports, to report the complications that occurred after the surgery, and to calculate and compare the frequency of complications for correction of MPL when using a new landmark.

\section{Materials and Methods}

We retrospectively evaluated 75 dogs (107 limbs) with MPL from July 2008 to May 2013. Information regarding the breed, age, sex, body weight, body condition score (BCS), lateral $v s$ bilateral, pre-operative grading, surgical techniques, removal of implants, concomitance with anterior cruciate ligament (ACL)
Correspondence: Mitsuhiro Isaka, Marble Veterinary Medical Center, 4-1-6 Ishikawa, Fujisawa, Kanagawa 252-0815, Japan.

Tel. +81.466.862.080 - Fax: +81.466.860.558.

E-mail: isakam7@gmail.com; mitsuhiro_isaka@ mcvj.com

Key words: veterinary, animals, surgery.

Contributions: the authors contributed equally.

Received for publication: 20 December 2013.

Revision received: 10 January 2014.

Accepted for publication: 21 January 2014.

This work is licensed under a Creative Commons Attribution NonCommercial 3.0 License (CC BYNC 3.0).

CC Copyright M. Isaka et al., 2014

Licensee PAGEPress srl, Italy

Veterinary Science Development 2014; 4:5251

doi:10.4081/vsd.2014.5251

rupture, re-luxation, re-operation, and rehabilitation was obtained from the medical records. MPL grading is shown in Table 1. The following surgical techniques were used: i) medial restraint release (release of the medial retinaculum), ii) lateral restraint reinforcement (imbrication of the joint capsule), iii) capsulolabral reconstruction, iv) trochlear grove deepening (block recession and trochlear chondroplasty), and/or v) tibial tuberosity transposition, by 2 surgeons (Dr. Isaka and Dr. Befu). Partial meniscectomy was performed if the meniscus was injured. The landmark for block recession was between the femoral trochlear notch and the attachment of the posterior cruciate ligament (PCL) (Figure 1). Subcutaneous robenacoxib (1 mg/kg) was used for preoperative pain control and continuous intravenous fentanyl $(1-5 \mathrm{~g} / \mathrm{kg} / \mathrm{h})$ was used intra operatively. Robert Jones bandages were applied for 10 days for post-operative management. Ice was applied to the surgical site [10 min, twice a day (b.i.d)] and Cefotax (20 $\mathrm{mg} / \mathrm{kg}$, b.i.d.) was administered intravenously for 3 days, and the skin suture was removed 10 days after the operation.

\section{Results}

We reviewed 75 cases (107 limbs) of MPL. As shown in Table 2, the following breeds were included in this study: Chihuahua $(\mathrm{n}=23)$, Pomeranian $(n=12)$, Yorkshire Terrier $(n=9)$, Toy Poodle ( $n=7)$, Papillon $(n=6)$, mixed breed $(\mathrm{n}=4)$, Maltese $(\mathrm{n}=3)$, Cavalier King Charles Spaniel $(n=2)$, Shiba $(n=2)$, Bulldog $(n=2)$, Yunbancyen $(\mathrm{n}=1)$, Miniature Dachshund $(n=1)$, BostonTerrier $(n=1), \quad$ Shetland 
Sheepdog $(\mathrm{n}=1)$, and West Highland White Terrier $(n=1)$. All evaluation variants are shown in Table 3 . The study sample included 33 males (castrated $n=13$ ) and 42 females (spayed $\mathrm{n}=21$ ). The median age was $53.3 \pm 35.9$ months (32-146 months); 13 cases were less than 12 months of age (17.3\%). The pre-operative BCSs were $1(\mathrm{n}=0), 2(\mathrm{n}=20), 3(\mathrm{n}=24), 4$ $(\mathrm{n}=24)$, and $5(\mathrm{n}=7)$. The body weight was $4.51 \pm 3.48 \mathrm{~kg}$ (1.34-23.0 kg); 71 cases $(94.7 \%)$ were less than $10 \mathrm{~kg}$. The MPL grades (each limb) were $\mathrm{G} 1(\mathrm{n}=1), \mathrm{G} 2(\mathrm{n}=18), \mathrm{G} 3(\mathrm{n}=78)$, and $\mathrm{G} 4(\mathrm{n}=10)$. Of the total cases, 32 were bilateral and 43 were unilateral (right $n=27$; left $n=16$ ). The specific surgical procedure (distal femoral osteotomy) was 3 stifles in Chihuahuas. Concurrent with ACL rupture was $16 / 107$ stifles (15.0\%) corrected with the overthe-top method or the extracapsular method in Papillons (5/6), Chihuahua (5/23), and so on. Hip dysplasia was found in only 2 limbs with MPL. Re-luxation and re-operation were noted in 3 out of 107 stifles (2.8\%) and in 0\%, respectively. However, re-luxation was not observed after January 2010. In addition, trochlear sulcoplasty was not performed in any of the reluxation cases.

\section{Discussion}

Numerous surgical techniques of trochlear groove deepening for canine MPL have been reported. In TBR, an osteochondral block is developed from the femoral trochlea by utilizing 2 abaxial osteotomies that are initiated slightly axial to the peak of each trochlear ridge and a transchlear (basilar) osteotomy that is angled approximately $90^{\circ}$ to the trochlear sulcus. The abaxial osteotomies are angled approximately $10^{\circ}$ from the sagittal plane so that they converge. The wedge is carefully removed once the 3 osteotomies are complete. Bone can be removed from the base of the recipient bed or from the basilar surface of the osteochondral block to achieve recession of the osteochondral block and the articular surface. The osteochondral block is replaced and is firmly pressed into the recipient bed to achieve a press fit. ${ }^{8}$ However, the success of this surgical technique depends on the experience of the surgeon. In our method, we used the attachment point of the PCL and the femoral trochlear notch as the landmark for trochlear groove deepening, and this technique provided good surgical results.

Bilateral MPL is common, occurring in 50\% (Arthurs et al., 2007) to $65 \%$ of cases. ${ }^{5,9}$ Bilateral cases were comprised $42.7 \%$ of the cases in our study, which is similar to the previous studies. The higher incidence of MPL in males compared with females is consistent with 3 previous reports. . $^{3-5}$
In a recent report, Linney et al. reported that the incidence of re-luxation and re-operation was $6.6 \%$ in total for 91 cases of MPL in dogs after non-block recession. ${ }^{2}$ In addition, Arthurs et al reported that the complication and reoperation rates after MPL surgery were 18\% and $8 \%$, recpectively. 1 Our surgical results were better compared to this previous study. Further, no re-luxation case was observed after 2010. Finally, trochear sulcoplasty was not performed for any of the re-luxation cases in our study. Interestingly, the incidence of concomi- tance with ACLt rupture was approximately $15.0 \%$ in our study, whereas the incidence was $41 \%$ in a previous study. ${ }^{3}$ Although this difference may be due to different breed distributions such a variation would not completely explain this disparity.

In a small breed study, the overall recurrence rate was $10 \%$ in Pomeranians. The outcome of surgery was considered good for grade II luxation with a $100 \%$ success rate. Recurrent MPL was diagnosed in approximately $11 \%$ of dogs with grade III and in $36 \%$ of dogs with

Table 1. Grades of patellar luxation.

\begin{tabular}{ll} 
Grade & Description \\
1 & $\begin{array}{l}\text { Patella can be manually luxated but returns tonormal position when released } \\
2\end{array}$ \\
$\begin{array}{l}\text { Patella luxates with stifle flexion or on manual manipulation and remains luxated until } \\
\text { stifle extension or manual replacement occurs }\end{array}$ \\
\hline 3 & $\begin{array}{l}\text { Patella luxated continually. Patella can be manually replaced but reluxates spontaneously } \\
\text { when manual pressure is removed }\end{array}$ \\
Patella luxated continually and cannot be manually replaced
\end{tabular}

Table 2. Breeds.

\begin{tabular}{lcc} 
Breed & Number of stifle & Case number \\
Chihuahua & 33 & 23 \\
Pomeranian & 16 & 12 \\
\hline Yorkshire Terrier & 15 & 9 \\
T. Poodle & 9 & 7 \\
\hline Papillon & 6 & 6 \\
Mix & 7 & 4 \\
\hline Maltese & 4 & 3 \\
Cavalier King Charles Spaniel & 3 & 2 \\
\hline Bulldog & 2 & 2 \\
Shiba & 3 & 2 \\
\hline Yunbancyen & 2 & 1 \\
Dachshund & 1 & 1 \\
\hline Boston terrier & 2 & 1 \\
Shetland sheep dog & 1 & 1 \\
\hline West highland white terrier & 1 & 1
\end{tabular}

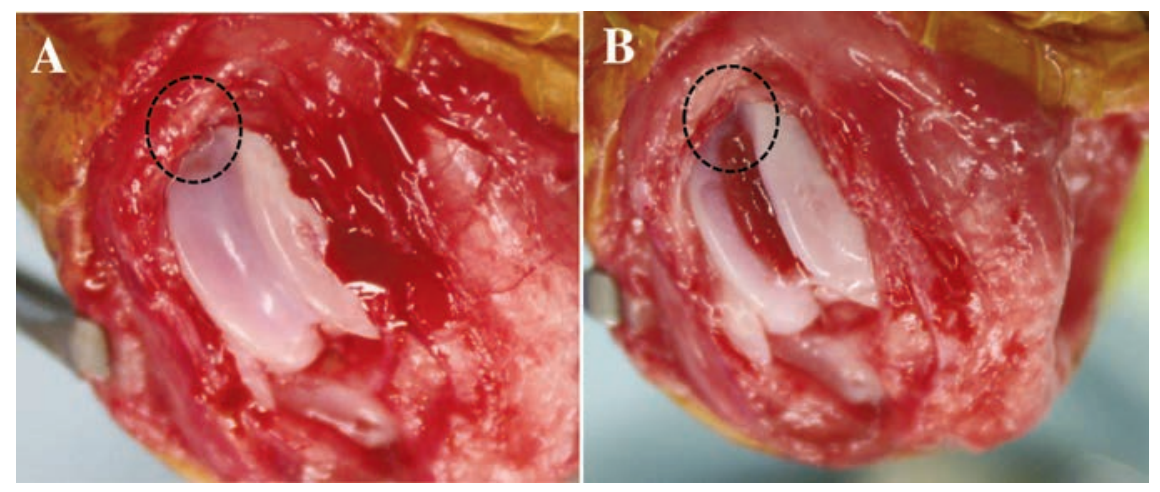

Figure 1. Surgical Landmark in pre-(A) and post-(B) block recession. 
sion in canine MPL, and that the use of this landmark leads to satisfactory surgical results.

\begin{tabular}{|c|c|c|c|c|}
\hline \multirow[t]{2}{*}{ Variable } & \multicolumn{2}{|c|}{ Number } & \multicolumn{2}{|c|}{ Percenatge } \\
\hline & Stifles & Cases & Stifles & Cases \\
\hline \multicolumn{5}{|l|}{ Body weight } \\
\hline$<10 \mathrm{~kg}$ & 102 & 71 & 97.1 & 94.7 \\
\hline$>10 \mathrm{~kg}$ & 5 & 4 & 2.9 & 5.3 \\
\hline \multicolumn{5}{|l|}{ Pre-operative BCS } \\
\hline 1 & - & 0 & - & 0 \\
\hline 2 & - & 20 & - & 26.7 \\
\hline 3 & - & 24 & - & 32.0 \\
\hline 4 & - & 24 & - & 32.0 \\
\hline 5 & - & 7 & - & 9.3 \\
\hline \multicolumn{5}{|l|}{ Age } \\
\hline$<12$ months & 16 & 11 & 15.2 & 14.7 \\
\hline$>12$ months & 91 & 64 & 84.8 & 85.3 \\
\hline \multicolumn{5}{|l|}{ Male } \\
\hline Intact and castrated & 44 & 33 & 41.1 & 44.0 \\
\hline Castrated & 14 & 13 & 13.1 & 17.3 \\
\hline \multicolumn{5}{|l|}{ Female } \\
\hline Intact and syapyed & 63 & 42 & 58.9 & 56.0 \\
\hline Spayed & 29 & 21 & 27.1 & 28.0 \\
\hline \multicolumn{5}{|l|}{ Lateral } \\
\hline Left and right & - & 43 & - & 57.3 \\
\hline Left hind-limb & - & 16 & - & 21.3 \\
\hline Right hind-limb & - & 27 & - & 36.0 \\
\hline Bilateral & - & 32 & - & 42.7 \\
\hline Trochlear sulcoplasty & 22 & - & 20.5 & - \\
\hline Trochlear sulcoplasty & 85 & - & 79.4 & - \\
\hline \multicolumn{5}{|l|}{ Pre-operative grading } \\
\hline G1 & 1 & - & 0.9 & - \\
\hline $\mathrm{G} 2$ & 18 & - & 16.8 & - \\
\hline G3 & 78 & - & 72.9 & - \\
\hline $\mathrm{G} 4$ & 10 & - & 9.3 & - \\
\hline Concomitant with ACL & 16 & 16 & 15.0 & 21.3 \\
\hline Re-luxation & 3 & 3 & 2.9 & $4^{* *}$ \\
\hline Re-operation & 0 & 0 & 0 & 0 \\
\hline Removal of implant & 33 & 20 & 30.8 & 26.7 \\
\hline Needs rehabilitation & 27 & 21 & 25.2 & 28.0 \\
\hline
\end{tabular}

**All re-luxation cases was without trochlear sulcoplasty.

grade IV luxation. ${ }^{10}$ Patellar luxation recurred in $2.6 \%$ of the dogs in our study, and this result is better than those reported by Alam (6\%), and Linney (19.8\%), who treated MPL with a combination of the lateral retinaculum imbrication and tibial tuberosity transposition techniques, but without the TBR technique. ${ }^{2,4}$ Thus, it appears that TBR is a definitive surgical method for MPL, resulting in a high surgical success rate. We agree with the previous recommendation that at least 1 corrective osteotomy such as tibial tuberosity transposition, femoral trochleoplasty, or tibial plateau leveling osteotomy with tibial axial re-alignment should be performed to reduce the patellar re-luxation rate. ${ }^{11}$ In our study, the treatment outcomes of grade II and III MPL after TBR were $100 \%$ and $88 \%$, respectively, and weight bearing and lameness improved following surgery.

TBR is a cartilage-sparing technique that was developed to achieve adequate trochlear depth and width, to maximize the preservation of hyaline articular cartilage, and to provide secure fixation of the osteochondral autograft. ${ }^{8}$ However, until now, a good landmark for TBR did not exist. In our TBR, we created a landmark between the attachment point of the PCL and femoral trochlear notch, which might not depend on the experience of the surgeon.

In our study, post-operative rehabilitation was required for approximately $25 \%$ of the cases. A protocol has been established for canine ACL rupture. ${ }^{12}$ To our knowledge, postoperative rehabilitation is definitely required for all canine ACL rupture. Thus, a rehabilitation protocol for canine MPL will be needed in the near future.

\section{Conclusions}

In conclusion, this retrospective study suggested that the area between the femoral trochlear notch and the attachment point of PCL is good surgical landmark for block reces-

\section{References}

1. Arthus GI, Langley-Hobbs SJ. Complications associated with corrective surgery for patellar luxation in 109 dogs. Vet Surg 2006;35: 559-66.

2. Linnney WR, Hammer DL, Shott S. Surgical treatment of medial patellar luxation without femoral trochlear groove deepening procedures in dogs: 91 cases (1998-2009). J Am Vet Med Assoc 2011;238:1168-72.

3. Campbell CA, Horstman CL, Mason DR, Evans RB. Severity of patellar luxation and frequency of concomitant cranial cruciate ligament rupture in dogs: 162 cases (20042007). J Am Vet Med Assoc 2010;236:887-91.

4. Alam MR, Lee JI, Kang HS, et al. Frequency and distribution of patellar luxation in dogs. 134 cases (2000 to 2005). Vet Comp Orthop Traumatol 2007;20:59-64.

5. Hayes AG, Boudrieau RJ, Hungerford LL. Frequency and distribution of medial and lateral patellar luxation in dogs: 124 cases (1982-1992). J Am Vet Med Assoc 1994;205: 716-20.

6. Johnson AL, Probst CW, Decamp CE, et al. Comparison of trochlear block recession and trochlear wedge recession for canine patellar luxation using a cadaver model. Vet Surg 2001;30:140-50.

7. Johnson AL, Broaddus KD, Hauptman JG, et al. Vertical patellar position in large-breed dogs with clinically normal stifles and largebreed dogs with medial patellar luxation. Vet Surg 2006;35:78-81.

8. Talcott KW, Goring TL, Haan JJ. Rectangular recession trochleoplasty for treatment of patellar luxation in dogs and cats. Vet Comp Orthop Traumatol 2000;13:39-43.

9. Arthurs GI, Langley-Hobbs SJ. Patellar luxation as a complication of surgical intervention for the management of cranial cruciate ligament rupture in dogs. A retrospective study of 32 cases. Vet Comp Orthop Traumatol 2007;20:204-10.

10. Wangdee C, Theyse LF, Techakumphu M, et al. Evaluation of surgical treatment of medial patellar luxation in Pomeranian dogs. Vet Comp Orthop Traumatol 2013;26:435-9.

11. Roy RG, Wallace LJ, Johnston GR, Wickstrom SL. A retrospective evaluation of stifle osteoarthritis in dogs with bilateral medial patellar luxation and unilateral surgical repair. Vet Surg 1992;21:475-9.

12. Jerre $\mathrm{S}$. Rehabilitation after extra-articular stabilization of cranial cruciate ligament rupture in dogs. Vet Comp Orthop Traumatol 2009;22:148-52. 